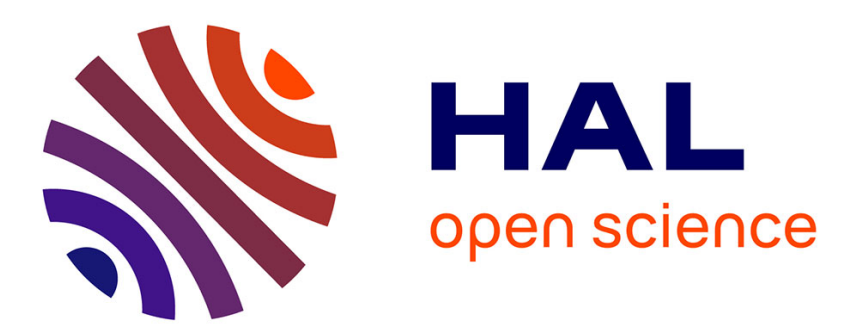

\title{
Nouvelles perspectives sur l'engagement des parties prenantes : enjeux, acteurs, recherches
}

\author{
André Sobczak, Ariane Berthoin Antal
}

\section{To cite this version:}

André Sobczak, Ariane Berthoin Antal. Nouvelles perspectives sur l'engagement des parties prenantes: enjeux, acteurs, recherches. Revue management \& avenir, 2010, (33), pp.117-126. 10.3917/mav.033.0116 . hal-00771163

\section{HAL Id: hal-00771163 \\ https://hal.science/hal-00771163}

Submitted on 11 Apr 2013

HAL is a multi-disciplinary open access archive for the deposit and dissemination of scientific research documents, whether they are published or not. The documents may come from teaching and research institutions in France or abroad, or from public or private research centers.
L'archive ouverte pluridisciplinaire HAL, est destinée au dépôt et à la diffusion de documents scientifiques de niveau recherche, publiés ou non, émanant des établissements d'enseignement et de recherche français ou étrangers, des laboratoires publics ou privés. 


\section{Nouvelles perspectives sur l'engagement des parties prenantes : enjeux, acteurs, recherches}

\section{par André Sobczak ${ }^{42}$ et Ariane Berthoin Antal ${ }^{43}$}

Pour explorer la pertinence et la fertilité du concept d'engagement des parties prenantes dans le contexte francophone, I'Institut pour la Responsabilité Globale a organisé en novembre 2009 un colloque à Audencia Nantes School of Management qui a réuni des chercheurs de différentes disciplines et champs du management. Si certains de ces chercheurs avaient déjà une connaissance approfondie de la théorie des parties prenantes et de leur engagement, nous avons aussi invité beaucoup d'autres pour porter un regard neuf sur cette théorie à travers le prisme de leurs propres travaux, par exemple dans le domaine de la fiscalité, du tourisme ou des relations de travail, et les cadres théoriques qu'ils mobilisent habituellement. Après ce colloque, nous avons invité certains participants à revoir leurs communications en vue de la préparation de ce dossier spécial de la Revue Management \& Avenir dont l'objectif est d'introduire les lecteurs aux principales réflexions liées à l'engagement des parties prenantes et d'illustrer la manière dont celles-ci peuvent contribuer dans le contexte français et belge aux débats académiques, à la pédagogie et aux pratiques dans et entre les organisations, créant ainsi les fondements pour d'autres développements futurs. ${ }^{44}$

Avant de présenter les différents articles de ce dossier spécial et leur contribution au débat sur l'engagement des parties prenantes pour la responsabilité globale, cette introduction cherche à mettre en évidence l'intérêt de cette thématique et à présenter les principaux enjeux dans ce domaine.

\section{Pourquoi s'intéresser à l'engagement des parties prenantes ?}

Au cours des cinq dernières décennies, les relations entre l'entreprise et la société ainsi que la manière dont les académiques analysent cette relation ont profondément changé. Partout dans le monde, les activités des entreprises font aujourd'hui l'objet d'une plus grande attention de la part d'un nombre croissant d'acteurs qui observent leurs impacts économiques, sociaux et environnementaux

\footnotetext{
42. André Sobczak, Audencia Nantes School of Management, Institut pour la Responsabilité Globale dans l'Entreprise asobczak@audencia.com

43. Ariane Berthoin Antal, Audencia Nantes School of Management, Wissenschaftszentrum Berlin für Sozialforschung (WZB), ABAntal@wzb.eu

44. Nous tenons à remercier Barbara Haddou pour son support autour du colloque et Annick Bertolotti pour son aide dans l'édition des contributions à ce dossier.
} 
de manière critique. Surtout, ces acteurs qui incluent notamment les médias, les pouvoirs publics, les organisations non gouvernementales, les consommateurs et les associations de riverains, ne se contentent plus uniquement d'évaluer les performances réalisées par les entreprises dans ces différents domaines et de leur demander des comptes sur le passé. Ils cherchent à influencer les processus même de décision dans les entreprises afin que celui-ci intègre leurs intérêts en amont, dès qu'il s'agit de définir les objectifs futurs. Par ailleurs, les entreprises doivent faire face à un éventail toujours plus large d'enjeux portés par les différents acteurs de la société et nécessitant souvent des solutions complexes, comme la lutte contre le changement climatique, la pauvreté ou les épidémies, mais aussi la promotion de l'égalité des chances ou la prise en compte des effets de l'urbanisation et des changements démographiques. Si la société pousse ainsi les entreprises à reconnaître des responsabilités nouvelles, leur définition et leur mise en œuvre impliquent souvent de formes de coopération innovantes entre tous les acteurs de la société, quels qu'en soit leur nationalité, leur taille et leur statut public ou privé.

Au cours de ces années, les chercheurs en management ont accompagné cette évolution, en analysant les relations entre l'entreprise et la société ou en formulant des préconisations sur la manière de faire vivre cette relation, voire en s'impliquant concrètement dans des projets de recherche-action avec des entreprises ou des représentants de la société civile. Aux Etats-Unis, après des premiers travaux par Berle et Means (1933), les chercheurs en management se sont surtout intéressés à cette relation entre l'entreprise et la société à partir des années 1960-1970. A la même époque, plusieurs expérimentations ont été menées en France et en Allemagne pour développer de nouveaux outils de reporting social comportant des indicateurs définis par différents acteurs de la société et devant conduire les dirigeants à chercher à atteindre des objectifs dépassant les seuls critères financiers (Preston, Rey et Dierkes, 1981). Après la période des années 1980-1990 qui ont été dominées dans la pratique et dans la recherche par l'intérêt pour la valeur actionnariale, l'approche du nouveau millénaire a mis en évidence le coût économique, social et environnemental d'un management concentré sur les intérêts d'un seul acteur, l'actionnaire, conduisant ainsi à la redécouverte de l'idée d'une responsabilité sociale des entreprises.

Dépassant cette fois-ci des initiatives ponctuelles, la responsabilité sociale se structure à partir de là en tant que courant académique, à la fois au niveau national, européen et international ${ }^{45}$, en créant des lieux de débats plus larges sur les relations entre l'entreprise et la société. En passant du terme de « relations entre l'entreprise et la société " vers celui de "responsabilité sociale des

45. Aux Etats-Unis, la structuration de ce courant a commencé dans les années 1970 avec la création d'un groupe d'intérêt, puis une division "Social Issues in Management » au sein de l'Academy of Management et le lancement de revues et de collections d'ouvrages spécialisées. Au niveau européen, le courant s'est structuré à partir de 2000 avec la création de la European Academy of Business in Society (EABIS), puis en France avec l'émergence du Réseau International de recherche sur les Organisations et le Développement Durable (RIODD). 


\section{Nouvelles perspectives sur l'engagement des parties prenantes : enjeux, acteurs, recherches}

entreprises ", on modifie cependant la perspective de l'analyse, mettant cette fois-ci l'entreprise, ses activités et ses modes de décision au cœur du débat. Cela s'est également traduit au niveau des cadres théoriques mobilisés par les chercheurs de ce courant. Les trois principaux cadres mobilisés dans ce domaine par les chercheurs en sciences de gestion (Gond et Mullenbach-Servayre, 2003) - la théorie des parties prenantes, la théorie contractualiste et la théorie institutionnaliste - s'articulent toutes autour de différentes manières de concevoir le rôle de l'entreprise et ses relations avec les autres acteurs.

Cela est vrai en particulier pour la théorie des parties prenantes développées par Freeman (1984) qui a, dès l'origine, fortement structuré le débat académique sur les relations entre l'entreprise et la société et encore plus celui sur la responsabilité sociale des entreprises. La définition même de ces parties prenantes est fondée sur le fait que les différents acteurs de la société influencent ou sont influencés par les activités de l'entreprise, comme si ces acteurs n'avaient pas d'existence propre indépendamment de l'entreprise. Cet aspect a été renforcé par le fait que, dans un premier temps, cette théorie a surtout été mobilisée par les chercheurs pour définir les principes permettant aux entreprises de " manager » leurs parties prenantes (Post, Preston et Sachs, 2002). Ces recherches visaient autant à amener les managers dans une perspective normative à reconnaître les intérêts et les apports potentiels des différentes parties prenantes qu'à mettre en place des pratiques et des procédures de management dans une perspective instrumentale.

Dans la mesure où les premières expériences et réflexions dans ce domaine ont été menées en Amérique du nord, ces recherches ont par ailleurs été fortement influencées par le contexte de ce continent. Or, aujourd'hui, la question des relations entre l'entreprise et la société se pose dans le monde entier, favorisant progressivement une meilleure prise en compte des différents contextes nationaux dans l'analyse des relations entre l'entreprise et la société. Au cours des dernières années, plusieurs recherches ont notamment souligné les spécificités des pratiques et réflexions sur la responsabilité sociale de entreprises en Europe (Habisch et al., 2005 ; Matten et Moon, 2008) et en particulier en France (Berthoin Antal et Sobczak, 2007) et en Allemagne (Berthoin Antal, Oppen et Sobczak, 2010). Mettant ainsi en évidence que les relations entre l'entreprise et la société peuvent se définir de différentes manières, ces recherches s'inscrivent dans un mouvement plus large visant à élargir les perspectives sur la responsabilité sociale des entreprises et la théorie des parties prenantes pour tenir compte des évolutions récentes dans les débats et les pratiques.

Une première série de recherches visent à questionner le fait que le concept de responsabilité sociale des entreprises place ces dernières au centre du débat. Ce point de vue se heurte au constat que la complexité des enjeux économiques, sociaux et environnementaux implique de plus en plus souvent l'élaboration 
de solutions collectives avec tous les acteurs quel que soit leur statut. Dans cette perspective, certains chercheurs suggèrent dès lors de faire évoluer le concept de responsabilité sociale des entreprises vers celui plus large de responsabilité sociale des organisations (Bento, 2009) pour souligner que toutes les organisations et non pas seulement les entreprises doivent agir de manière responsable. Nous proposons d'aller encore plus loin et pour retenir la notion de responsabilité globale (Berthoin Antal et Sobczak, 2004) qui met aussi mieux en évidence que les intérêts à prendre en compte ne sont pas uniquement sociaux, mais au moins aussi environnementaux et économiques et qu'ils doivent être abordés de manière transversale et dans un contexte international ${ }^{46}$.

Liée à ces premiers travaux, une deuxième série de recherches vise à faire évoluer la manière d'analyser les relations entre l'entreprise et ses parties prenantes pour tenir compte du fait que ces dernières se sont multipliées et ont gagné en force, notamment en mobilisant souvent avec succès la communication par internet pour partager des informations ou exercer la pression. II semble en effet évident que certaines de ces parties prenantes, comme les organisations non gouvernementales ne comptent pas se laisser " manager » par les dirigeants d'entreprise. Dès lors, certains chercheurs suggèrent de faire évoluer le concept de management des parties prenantes vers celui de leur engagement (Andriof et al., 2002a, 2003), traduisant ainsi le fait que quelques entreprises innovantes ont évolué d'un management réactif des enjeux sociaux et environnementaux qui se limitait à respecter les normes vers des initiatives plus proactives et interactives permettant de traiter ces enjeux de manière plus efficace par des stratégies d'engagement de leurs réseaux de parties prenantes (Andriof et Waddock, 2002 : 41-42).

Lorsque l'on introduit un nouveau terme dans le débat, comme celui d'engagement des parties prenantes, il est toujours utile de commencer par l'analyse de sa signification. Le dictionnaire anglais propose différentes situations dans lesquelles on évoque l'engagement, comme un mariage ou un accord, mais également une lutte. Le dictionnaire français contient une liste similaire de significations, comme le « fait de prendre parti sur les problèmes politiques et sociaux par son action et ses discours » ou un "combat de courte durée et localisée ॥. II précise que le terme d'engagement renvoie aussi bien à l'acte par lequel on s'engage à accomplir quelque chose qu'à l'action d'engager quelqu'un. Par ailleurs, il explique que le terme peut recouvrir des significations très spécifiques dans certains contextes professionnels, comme par exemple « l'ensemble des obligations envers des tiers » en comptabilité, " l'acte par lequel le roi de France concédait à un engagiste des terres du domaine royal » en histoire ou alors « le franchissement du plan du détroit supérieur du bassin maternel par le plus grand diamètre de la présentation fœtale » en médecine. Il est par conséquent essentiel

\footnotetext{
46. L'idée de la transversalité des enjeux qui dépassent la seule dimension sociale est aussi évoquée dans les travaux préconisant le recours à la notion de "responsabilité des entreprises » (Waddock et Smith, 2000), une notion qui, contrairement à celle de responsabilité globale, maintient cependant l'entreprise au centre des débats.
} 


\section{Nouvelles perspectives sur l'engagement des parties prenantes : enjeux, acteurs, recherches}

de s'accorder sur le contenu que nous donnons à la notion d'engagement des parties prenantes en matière de responsabilité globale.

Greenwood (2007) s'oppose à une utilisation trop vague ou trop large du terme et suggère de définirl'engagement des parties prenantes comme un ou des processus de consultation, de communication, de dialogue et d'échange (2007 : 321-322). Vu la dynamique des thématiques et des acteurs, nous pensons que ce processus implique aussi une capacité d'apprentissage et partage de connaissances. Ainsi nous proposons de définir l'engagement des parties prenantes comme la création active de relations d'apprentissage avec les différentes parties prenantes afin de trouver des solutions aux enjeux économiques, sociaux et environnementaux.

La littérature académique utilise cette référence au processus le plus souvent de manière positive, au moins implicitement, en soulignant par exemple que « le processus d'engagement crée un contexte dynamique d'interaction, de respect mutuel, de dialogue et de changement, non pas un management unilatéral des parties prenantes » (Andriof et al., 2002b : 9, italique dans le texte, notre traduction). Cette manière de voir pourrait laisser penser que l'engagement des parties prenantes se traduit toujours par des interactions harmonieuses entre égaux. II semble dès lors utile de rappeler qu'aussi bien le dictionnaire anglais que le dictionnaire français évoque également une dimension conflictuelle de l'engagement. Certains chercheurs soulignent donc, à juste titre, que « dans les interactions intensives qu'implique l'engagement des parties prenantes, le conflit est presque inévitable » (Rahman et al., 2003 : 11, notre traduction).

Cela est d'autant plus vrai que le processus d'engagement des parties prenantes concerne un panel d'acteurs toujours plus large dont la composition change en permanence, obligeant les organisations à interagir en même temps avec des multiples parties prenantes dont les valeurs, les intérêts, mais également les langues et les styles de communication diffèrent souvent de manière importante. L'engagement des parties prenantes suppose aussi la capacité d'interagir de manière bilatérale et multilatérale, puisque les organisations sont insérées dans des réseaux de parties prenantes qui peuvent s'engager ensemble. Par conséquent, une cartographie des parties prenantes n'est pas transposable d'un contexte à un autre. La réalisation d'une telle cartographie est un processus qui n'est jamais finie et qui doit être suffisamment affinée pour identifier les différentes catégories de parties prenantes et leur donner un visage avec lequel il est possible de s'engager concrètement. La dimension dynamique de la notion d'engagement met par ailleurs en évidence le fait que les enjeux sur l'agenda des parties prenantes peuvent changer rapidement. De nouveaux enjeux, parfois plusieurs en même temps, peuvent ainsi devenir prioritaires pour certaines parties prenantes, obligeant les praticiens et les chercheurs à organiser une veille permanente sur les évolutions dans les catégories de parties prenantes et leurs intérêts. 


\section{Comment ce dossier spécial peut-il enrichir le débat sur ces enjeux ?}

Pour analyser l'engagement des parties prenantes pour la responsabilité globale, ce dossier spécial est organisé en quatre parties. La première, vise à dresser un bilan de l'évolution des recherches dans le domaine de l'engagement des parties prenantes au cours des cinq dernières décennies, tout en mettant aussi en évidence les nouveaux enjeux et les perspectives d'avenir. Après cet article d'introduction, David Bevan et Patricia Werhane proposent une analyse critique de la théorie des parties prenantes qui retrace l'évolution des idées lancées depuis 1984 par R. Edward Freeman, notamment dans la perspective de l'éthique de l'entreprise. Ils reconnaissent en particulier la nécessité de dépasser une vision actuellement trop centrée sur l'entreprise et de donner un visage aux différentes catégories de parties prenantes. Dans le troisième article de cette première partie, Samuel Mercier nous rappelle que les idées et les débats son en fait bien plus anciennes, remontant aux années 1930 aux Etats-Unis. II revient sur les ambitions des défenseurs de la théorie des parties prenantes pour mieux mettre en évidence ses faiblesses. II explique ainsi ce que la mobilisation de ce cadre théorique peut et ce qui ne peut pas permettre de comprendre ou de changer dans le management des entreprises. Cette première partie présente le cadre et montre le besoin de développer de nouvelles perspectives sur l'engagement des parties prenantes pour la responsabilité globale, un défi que les auteurs des trois parties suivantes relèvent.

La deuxième partie contient une série d'articles qui analysent le processus d'identification et de représentation des différentes parties prenantes d'une organisation et de leurs intérêts, en soulignant l'importance de s'adapter à un contexte qui change en permanence et fait émerger de nouveaux acteurs. Cette étape semble essentielle pour permettre à l'organisation de définir ensuite une stratégie et des actions adaptées. Dans le premier article de cette partie, Carine Girard et André Sobczak cherchent à répondre au défi de la diversification des enjeux et des intérêts des parties prenantes, en proposant une nouvelle cartographie des parties prenantes qui permet de distinguer plusieurs souscatégories, en fonction de leur degré d'engagement à l'égard de l'organisation et à l'égard des valeurs de la responsabilité sociale. En décidant de retenir ce double degré d'engagement pour situer les différentes parties prenantes, cet article souligne la nécessité de renouveler régulièrement ce processus d'identification pour mesurer l'impact des pratiques de management sur l'engagement, mais aussi pour tenir compte du fait de l'émergence de nouvelles catégories ou sous-catégories de parties prenantes. Le deuxième article de cette partie décrit justement des processus d'émergence de nouvelles catégories de parties prenantes, en plaçant au centre de l'analyse les stratégies et actions de ces parties prenantes plutôt que l'entreprise qui les influence ou qui est influencée par celles-ci. L'article de Jean-Luc Rossignol porte sur l'administration fiscale et montre comment celle-ci modifie progressivement son comportement vis-à-vis 


\section{Nouvelles perspectives sur l'engagement des parties prenantes : enjeux, acteurs, recherches}

des entreprises en ne se limitant plus au rôle d'une autorité étatique qui impose, mais en se définissant de plus en plus comme une partie prenante qui cherche à convaincre, tout en s'appuyant sur les nombreuses informations et pouvoirs dont elle dispose. Dans le dernier article de cette partie, Jean-Paul et Nicolas Minvielle montrent les difficultés de défendre les intérêts des parties prenantes qui n'ont pas de voix, comme l'environnement ou la culture locale menacés par l'activité du tourisme au Sahara et encore peu défendus par les habitants qui peuvent en bénéficier à court terme. Les auteurs posent également la question des conséquences à tirer de l'efficacité de l'engagement de certaines parties prenantes pour la responsabilité globale. Ils s'interrogent ainsi si, face à une telle situation, les parties prenantes ne devraient pas se désengager.

La troisième partie de ce dossier spécial explore les différentes formes et motivations pour engager les parties prenantes. Dans un premier article, Yvan Barel et Sandrine Frémeaux analysent les raisons de l'engagement des personnes chargées de promouvoir la diversité dans les organisations. Ils montrent que le seul argument économique n'est pas suffisant pour comprendre les actions de cette catégorie de parties prenantes dans la mesure où elles sont très fortement liées à leur volonté de donner un sens à leur travail. L'article de Christophe Germain et Stephen Gates porte sur les outils de mesure mis en place par les entreprises en matière de responsabilité globale, en particulier pour communiquer les objectifs et les résultats auprès des parties prenantes, et sur leurs liens avec la stratégie des entreprises. Les résultats de cette enquête semble indiquer que très peu d'entreprises ont mis en place des mesures de la performance sociale et environnementale reliées à leur stratégie, ce qui pourrait signifier qu'elles ne cherchent pas réellement à engager les parties prenantes et se limitent à communiquer sur la responsabilité globale sans modifier leur stratégie. Cependant, des interprétations plus optimistes sont possibles, car l'absence de lien peut aussi s'expliquer par le fait que l'engagement des parties prenantes ne s'est pas encore traduite par une modification des outils de pilotage, ou simplement par le fait que l'engagement des parties prenantes et la mesure de la performance globale relèvent d'autres équipes dans l'entreprise que le pilotage et la stratégie de l'entreprise. La troisième partie est conclue par l'article de Martin Fougère qui y analyse une expérience d'enseignement dans une école de management visant à mieux préparer les étudiants et futurs décideurs dans les entreprises ou d'autres organisations à intégrer les intérêts des différentes parties prenantes. En s'appuyant sur un jeu de rôles largement inspiré d'un conflit réel entre une entreprise et ses différentes parties prenantes, cette expérience pédagogique permet de mettre en évidence la dimension politique de la responsabilité globale et des relations entre les parties prenantes.

La quatrième et dernière partie de ce dossier spécial contient plusieurs articles qui explorent de nouveaux cadres théoriques ou de nouvelles méthodologies 
de recherche pour analyser l'engagement des parties prenantes, repositionnant donc le débat théorique sur les parties prenantes et ouvrant de nouvelles perspectives pour l'analyse des relations entre l'entreprise et la société. Dans le premier article de cette partie, Cathy Krohmer, Christine Naschberger et André Sobczak analysent le processus d'émergence des réseaux de promotion de la diversité comme nouvelle catégorie de parties prenantes des entreprises qui mettent en œuvre des actions dans ce domaine. Ils proposent d'enrichir l'analyse de ce processus, en mobilisant un cadre théorique peu utilisé dans le domaine des relations entre l'entreprise et la société, à savoir la théorie de la régulation sociale (Sobczak, 2010). Toujours dans le domaine de la diversité, l'article de Manal El Abboubi et Annie Cornet porte sur la labellisation des démarches des entreprises dans ce domaine. Les auteurs s'appuient sur la théorie de l'acteur-réseau pour enrichir l'étude des relations entre l'entreprise et la société et pour démontrer que l'engagement d'une partie prenante - en l'occurrence les organisations syndicales - peut être imposé par les pouvoirs publics, dès lors que ceux-ci décident de ne valoriser que les démarches ayant été validées par cette partie prenante. En conclusion de cette partie et de ce dossier spécial, Virginie Xhauflair et François Pichot s'interrogent sur les conditions qui peuvent favoriser l'émergence et la viabilité dans le temps d'un compromis entre différentes parties prenantes d'une situation. Analysant une étude de cas, les auteurs montrent l'intérêt une méthodologie de recherche de nature abductive, fondée sur la stimulation et l'accompagnement de compromis émergents, et la collaboration avec les parties prenantes. Ils mettent ainsi en évidence une possibilité d'engagement des chercheurs qui constituent également une catégorie de parties prenantes des organisations avec lesquelles ils collaborent ou dans lesquelles ils mènent leurs travaux.

\section{Pourquoi multiplier les perspectives?}

Rédigés par des chercheurs européens et américains venant d'horizons et de disciplines divers, les différents articles rassemblés dans ce dossier spécial peuvent contribuer à faire évoluer le débat académique et les pratiques dans le domaine des relations entre l'entreprise et la société. Ensemble, ils illustrent tout d'abord la diversité des parties prenantes et des formes d'engagement. Alors que la très grande majorité des recherches se limitent à analyser les intérêts, stratégies et actions des catégories de parties prenantes les plus connues, comme les actionnaires, les salariés, les clients, les fournisseurs ou plus récemment les organisations non gouvernementales, ce numéro ajoute des études sur les sociétaires, les organisations syndicales ou l'administration fiscale ; surtout il met en évidence que de nouvelles catégories de parties prenantes continuent toujours à émerger ou à être reconnues en tant que telles. Les articles permettent de donner un contenu concret aux intérêts de ces différentes catégories de parties prenantes, à leurs stratégies, à leurs modes d'action et à leurs pratiques d'engagement plutôt que de se cantonner comme la majorité des recherches 


\section{Nouvelles perspectives sur l'engagement des parties prenantes : enjeux, acteurs, recherches}

dans ce domaine à analyser la manière dont les managers de l'entreprise gèrent les relations avec elles.

En élargissant ainsi le champ de l'analyse, les articles de ce dossier spécial illustrent la diversité et la complexité des formes d'engagement des parties prenantes. Ils révèlent une dimension humaine à tout engagement, donnant un visage à chaque partie prenante considérée. Plusieurs articles montrent ainsi que l'engagement passe souvent par des phases de conflit plus ou moins ouvert, mais indiquent également des pistes qui peuvent permettre aux acteurs de transformer l'énergie du conflit pour entrer ensuite dans une relation plus constructive permettant d'apprendre ensemble pour trouver des compromis fondés sur des solutions innovantes. D'autres articles insistent sur la dimension affective de l'engagement des parties prenantes dans la responsabilité globale qui repose souvent sur la passion des individus, leur empathie et leur capacité d'influence ou d'entraînement des autres acteurs. En reconnaissant et en analysant ces différentes dimensions de l'engagement, ce dossier spécial vise à changer le regard sur les relations entre l'entreprise et la société. Nous y voyons à la fois un défi et une opportunité non seulement pour les acteurs dans les organisations mais aussi pour les chercheurs, enseignants et étudiants venant de disciplines diverses: sauront-ils s'engager réellement à ouvrir de nouvelles perspectives et à faire évoluer ces relations dans l'esprit de la responsabilité globale?

\section{Références}

Andriof J., Waddock S., Husted B. \& Rahman, S.S. (eds.) (2002a), Unfolding Stakeholder Thinking. Vol. 1: Theory, Responsibility and Engagement, Greenleaf Publishing, Sheffield UK.

Andriof J., Waddock S., Husted B. \& Rahman S.S., (2002b), « Introduction », in J. Andriof, S. Waddock, B. Husted \& S.S. Rahman (eds.), Unfolding Stakeholder Thinking. Vol. 1: Theory, Responsibility and Engagement, Greenleaf Publishing, Sheffield UK, p. 9-16.

Andriof J., Waddock S. (2002), "Unfolding Stakeholder Engagement ", in J. Andriof, S. Waddock, B. Husted \& S.S. Rahman (eds.), Unfolding Stakeholder Thinking. Vol. 1: Theory, Responsibility and Engagement, Greenleaf Publishing, Sheffield UK, p. 19-42.

Andriof J., Waddock S., Husted B., Rahman S.S. (eds.) (2003), Unfolding Stakeholder Thinking. Vol 2: Relationships, Communication, Reporting and Performance, Greenleaf Publishing, Sheffield UK.

Bento L. (2009), « De la CSR Corporate Social Responsibility à la RSO Responsabilité Sociale des Organisations. Quelques apports à la réflexion », Management \& Avenir, No 23, p. 73-82.

Berle A. A., Means G. (1932), The Modern Corporation and the Private Property, McMillan, New York.

Berthoin Antal A. \& Sobczak A. (2004), « Beyond CSR: Organisational Learning for Global Responsibility », Journal of General Management, Vol. 30, n² 2, p. 77-98.

Berthoin Antal, A. \& Sobczak, A. (2007), « Corporate Social Responsibility in France: A Mix of National Traditions and International Influences », Business \& Society, Vol. 46, $n^{\circ}$ 1, p. 9-32.

Berthoin Antal A., Oppen M. \& SobczakA. (2010), « (Re)discovering the social responsibility of business in Germany », Journal of Business Ethics, pre-print edition available at http:// 


\section{management \& avenir}

www.springerlink.com/content/p0189k01766rk577/.

Freeman, R.E. (1984), Strategic Management: A Stakeholder Approach. Pitman, Boston. Gond J.-P. \& Mullenbach-Servayre A. (2003), " Les fondements théoriques de la responsabilité sociétale de l'entreprise », Revue des Sciences de Gestion, n²05, p. 93116.

Greenwood M. (2007), « Stakeholder Engagement: Beyond the Myth of Corporate Responsibility », Journal of Business Ethics, Vol. 74, p. 315-327.

Habisch A., Jonker J., Wegner M. \& Schmidpeter R. (eds.) (2005), Corporate Social Responsibility Across Europe, Springer, Berlin.

Matten D. \& Moon J. (2008), « 'Implicit' and 'Explicit' CSR: A conceptual framework for a comparative understanding of corporate social responsibility », Academy of Management Review, Vol. 33, n², 404-424.

Post J.E., Preston L.E. \& Sachs, S. (2002), Redefining the corporation: Stakeholder management and organizational wealth, Stanford University Press, Stanford.

Preston L.E., Rey F., Dierkes M. (1981), « Comparing Corporate Social Performance: Germany, France Canada, and the U.S. », in S.P. Sethi \& C.L. Swanson (Eds.), Private Enterprise and Public Purpose, John Wiley \& Sons, New York, 117-128.

Rahman S.S., Waddock S., Andriof J. \& Husted B. (2003), « Introduction », in J. Andriof, S. Waddock, B. Husted \& S.S. Rahman (Eds.) Unfolding Stakeholder Thinking. Vol 2: Relationships, Communication, Reporting and Performance, Greenleaf Publishing, Sheffield, UK, p. 9-12.

Sobczak A. (2010), Multiplier les perspectives pour enrichir l'analyse des pratiques de responsabilité sociale des entreprises, Habilitation à Diriger des Recherches, Université Paris 12.

Waddock S. \& Smith N. (2000), « Relationships: The Real Challenge of Corporate Global Citizenship », Business and Society Review, Vol. 105, n 1, 47-62. 
Copyright of Revue Management et Avenir is the property of Management Prospective Editions and its content may not be copied or emailed to multiple sites or posted to a listserv without the copyright holder's express written permission. However, users may print, download, or email articles for individual use. 
Copyright of Revue Management et Avenir is the property of Management Prospective Editions and its content may not be copied or emailed to multiple sites or posted to a listserv without the copyright holder's express written permission. However, users may print, download, or email articles for individual use. 
Copyright of Revue Management et Avenir is the property of Management Prospective Editions and its content may not be copied or emailed to multiple sites or posted to a listserv without the copyright holder's express written permission. However, users may print, download, or email articles for individual use. 